1 Split-HaloTag ${ }^{\circledR}$ Imaging Assay for Sophisticated Microscopy of Protein-Protein

\title{
Interactions in planta
}

3 Rieke Minner-Meinen ${ }^{1}$, Jan-Niklas Weber ${ }^{1}$, Andreas Albrecht ${ }^{2}$, Rainer Matis ${ }^{2}$, Maria

4 Behnecke $^{1}$, Cindy Tietge ${ }^{1}$, Stefan Frank ${ }^{1}$, Jutta Schulze ${ }^{1}$, Henrik Buschmann ${ }^{3}$, Peter Jomo

5 Walla ${ }^{2}$, Ralf-R. Mendel ${ }^{1}$, Robert Hänsch ${ }^{1,4 *}$ and David Kaufholdt ${ }^{1}$

r.meinen@tu-bs.de,jan-niklas.weber@tu-bs.de, andreas.albrecht@tu-bs.de,r.matis@tubs.de, maria.behnecke@dsmz.de,cindy.oettel@tu-bs.de, stefan.frank@tu-bs.de, jutta.schulze@tu-bs.de, Henrik.buschmann@biologie.uni-osnabrueck.de,p.walla@tu-bs.de, r.mendel@tu-bs.de,r.haensch@tu-bs.de,d.kaufholdt@tu-bs.de

${ }^{1}$ Institut für Pflanzenbiologie, Technische Universität Braunschweig, Humboldtstrasse 1, D38106 Braunschweig, Germany

2Institut für Physikalische und Theoretische Chemie, Technische Universität Braunschweig, Hagenring 30.023c, D-38106 Braunschweig, Germany

${ }^{3}$ Botany Department, Universität Osnabrück, Barbara Str. 11, 49076 Osnabrück Germany

${ }^{4}$ Center of Molecular Ecophysiology (CMEP), College of Resources and Environment, Southwest University, Tiansheng Road No. 2, 400715 Chongqing, Beibei District, P.R. China

*author for correspondence:Tel:

$+49-(0) 531-391-5867$

Fax: $\quad+49-(0) 531-391-8128$

E-Mail: r.haensch@tu-bs.de

Date of submission: 2020-02-02

Word count total: 5918

Number of Tables: 0

Number of Supplementary Tables: 2

Number of Figures: 3

Number of Supplementary Figures: 2

Running title: Split-HaloTag ${ }^{\circledR}$ Imaging Assay 


\section{Abstract}

An ever-increasing number of intracellular multi-protein networks have been identified in plant cells. Split-GFP based protein-protein interaction assays combine the advantages of in vivo interaction studies in a native environment with additional visualisation of protein complex localisation. Due to its simple protocols, it has become one of the most frequently used methods. However, standard fluorescent proteins entail several drawbacks for sophisticated microscopy.

35 With the HaloTag ${ }^{\circledR}$ system, these drawbacks can be overcome as this reporter forms covalent irreversible bonds with synthetic photostable fluorescent ligands. Dyes can be used in adjustable concentrations and are suitable for advanced microscopy methods. Therefore, we established the Split-HaloTag ${ }^{\circledR}$ imaging assay in plants which is based on the reconstitution of a functional HaloTag ${ }^{\circledR}$ protein upon protein-protein interaction and subsequent covalent binding of an added fluorescent ligand. Its suitability and robustness were demonstrated using well-characterised interactions as an example for protein-protein interaction at cellular structures: the molybdenum cofactor biosynthesis complex anchoring to filamentous actin. Additionally, a specific interaction was visualised with subdiffractional polarisation microscopy in a more distinctive manner as example for sophisticated imaging. Split-GFP and Split-HaloTag ${ }^{\circledR}$ can complement one another as Split-HaloTag ${ }^{\circledR}$ represents an alternative option and an addition to the large toolbox of in vivo methods. Therefore, this promising new Split-HaloTag ${ }^{\circledR}$ imaging assay provides a unique and sensitive approach for more detailed characterization of protein-protein interaction with specific microscopic techniques such as 3D-imaging, single molecule tracking and super-resolution microscopy. 


\section{Keywords}

52 advanced microscopy, cytoskeleton, photostable fluorescent dyes, Gateway cloning,

53 Molybdenum cofactor biosynthesis complex, polarisation microscopy, protein-protein

54 interaction, Split-HaloTag ${ }^{\circledR}$ imaging assay

\section{Abbreviations}

56 BiFC: bimolecular fluorescence complementation; diAcFAM: diacetyl derivative of fluorescein;

57 FP: fluorescent protein; SPoD: Super-resolution by polarisation demodulation; TMR:

58 Tetramethylrhodamine 


\section{Introduction}

An ever-increasing number of protein networks has been identified in plants (Zitnik et al., 2019). Therefore, understanding the cellular biology of substrate channelling pathways requires the characterisation of protein-protein interactions (PPIs) in their native environment. A broad spectrum of in vivo methods has been employed to analyse PPIs such as bimolecular fluorescence complementation (BiFC) belonging to the group of protein fragment complementation assays (Struk et al., 2019). Basically, two non-fluorescent reporter fragments of a fluorescent protein (FP) are fused genetically to putative interaction partners and an interaction between the two allows formation of a bimolecular fluorescent complex (Kerppola, 2008). Consequently, BiFC not only allows detection of PPIs but also visualisation and localisation of the protein complex (Bhat et al., 2006). Furthermore, using FPs results in fluorescence signals without an invasive insertion of exogenous chemical compounds into the cell. Therefore, due to its simple protocols BiFC has become one of the most popular and frequently used method to study PPIs in plant cells (Kudla \& Bock, 2016).

Conventional light and fluorescence microscopy are diffraction-limited to a resolution limit of approx. $200 \mathrm{~nm}$ in the lateral (x-y) and about $600 \mathrm{~nm}$ in the axial (z) direction (Cremer and Masters, 2013). Many subcellular structures are smaller which hampers their detailed observation (Huang et al., 2009). To circumvent these restrictions, advanced fluorescence imaging methods such as single molecule detection, subdiffractional polarisation imaging or super-resolution microscopy (SRM) techniques have been developed to improve the resolution and to allow studying molecular processes more detailed (Moerner and Kador 1989; Orrit and Bernard, 1990; Bode et al., 2008; Holleboom et al., 2014; Liao et al., 2010/2011; Godin et al., 2014; Loison et al., 2018; Camacho et al., 2019). However, for such advanced imaging techniques fluorescent dyes with high stability and brightness are needed (Banaz et al., 2018), which is hard to realise by standard FPs as they show low quantum efficiency, blinking behaviour, a high photobleaching rate during long-term observations (Reck-Petersen et al., 2006), photoswitching (Morisaki and McNally, 2014) as well as the tendency to form oligomers (Miyawaki et al., 2003).

Self-labelling enzyme tags such as the HaloTag ${ }^{\circledR}$ have been shown to overcome these drawbacks of FPs and are suitable for such microscopy methods and super-resolution imaging 
89 (Grimm et al., 2015; Lee et al., 2010). The HaloTag ${ }^{\circledR}$ System (Promega, 90 https://www.promega.de/) is based on the bacterial haloalkane dehalogenase DhaA (EC 91 3.8.1.5) from Rhodococcus rhodochrous (Liss et al., 2015). The tag was modified to form covalent irreversible bonds with synthetic chloralkane ligands (Los et al., 2008; England et al., 2015). This covalent bond is formed rapidly under physiological environments and remains intact even under stringent conditions (Los and Wood, 2007). In addition, HaloTag ${ }^{\circledR}$ proteins keep their monomeric structure, so the tag will not lead to oligomerisation of protein fusion partners (Banaz et al., 2018). Numerous ligands are available for the HaloTag ${ }^{\circledR}$ including different fluorescent dyes with extended spectral range, photostability and membrane permeability such as the red fluorescent rhodamine derivative TMR (tetramethylrhodamine), the green fluorescent Oregon Green or the yellow fluorescent diacetyl derivative of fluorescein DiAcFAM. Furthermore, dyes can be varied in their dosages to either label all or only a few molecules which is needed for single-molecule tracking approaches. Reck-Peterson and colleagues (2006) for example used HaloTag ${ }^{\circledR}$ and a TMR ligand successfully to label dynein in sea urchin axonemes and tracked single molecules with a precision of a few nanometres to reveal dynein's stepping behaviour at microtubules. Moreover, several organic fluorophores especially for live-cell labelling and subsequent imaging were recently developed with photoactivatable properties (Lee et al., 2010) as well as with improved quantum efficiency and superior brightness while retaining excellent cell permeability (Grimm et al., 2015).

In 2012, Ishikawa and colleagues identified several split points within the HaloTag ${ }^{\circledR}$ protein and demonstrated its reconstitution ability. These results gave rise to the idea of establishing

111 the Split-HaloTag ${ }^{\circledR}$ imaging assay in planta as the usability of the HaloTag ${ }^{\circledR}$ imaging system in 112 plants has been shown previously (Lang et al., 2006). This new Split-HaloTag ${ }^{\circledR}$ approach is particularly useful for characterising the assembly protein complexes at structural elements

114 including cell membranes or the cytoskeleton. The possibility of using these microscopy 115 techniques will enable to study local formation of a given complex with improved details compared to BiFC and conventional confocal laser scanning microscopy. Single-molecule

117 tracking approaches with low concentrated fluorescent dye will enable the tracing of complex 118 mobility at the cytoskeleton or inside the membrane system. In this study, to establish the new Split-HaloTag ${ }^{\circledR}$ imaging assay we used the previously described anchoring of the molybdenum cofactor biosynthesis complex via molybdenum insertase $\mathrm{Cnx} 1$ to filamentous 
121 actin (Kaufholdt et al., 2017). In this way, we demonstrate the advantages of this assay for

122 imaging of in vivo protein-protein interactions via advanced microscopy.

\section{Material and Methods}

124 Cloning of Split-HaloTag ${ }^{\circledR}$ Gateway destination vectors

125 The optimised HaloTag ${ }^{\circledR}-7$ sequence $(298$ amino acids $)$ from Promega

126 (https://www.promega.de/) was genetically split on position 155/156 aa into the N-terminal

127 fragment “NHalo" (aa 1-155) and the C-terminal fragment "CHalo" (aa 156-298) according to

128 the initial experiment of Ischikawa and colleagues (2012). In order to create Split-HaloTag ${ }^{\circledR}$

129 GATEWAY ${ }^{\circledR}$ destination vectors, enabling C-terminal Split-HaloTag ${ }^{\circledR}$ reporter fusion, the binary 130 destination vectors pDest- Cluc-GW and pDest-GW-Cluc were used (Gehl et al., 2011). PCR-

131 Primers were designed to fuse specific restriction enzyme recognition sequences at both 132 reporter fragments (Table S1). After amplification, Cluc fragments were exchanged by 133 restriction and ligation for Nhalo or Chalo residues using the restriction sites Xbal and Spel (N134 terminal) and Xhol/Sacl (C-terminal), respectively (restriction enzymes purchased by Thermo 135 Fischer Scientific (https://www.thermofisher.com)), to create pDest-Nhalo-GW, pDest-GWNhalo, pDest-Chalo-GW and pDest-GW-Chalo (Table S2).

\section{Expression vectors}

138 Coding sequences of Cnx6 (AT2G43760), Cnx7 (AT4G10100) and Map65 (amino acids 340139 587; AT5G55230) were fused to Split-HaloTag ${ }^{\circledR}$ fragments via a two-step fusion PCR with Phusion-Polymerase purchased from Thermo Fischer Scientific

141 (https://www.thermofisher.com). For the first PCR, each single cDNA and reporter fragment 142 was created with an overlapping sequence to each, which enable assembly of fusion 143 constructs (used primers listed in Table S1). For the second step, the products of the first PCR 144 were assembled due to the overlapping matching sequences and then amplified a one single 145 fragment. This attB-site flanked constructs were subcloned via BP-reaction into the Donor 146 vector pDONR/Zeo to create entry vectors. Recombining these into pK7WG2 (Karimi et al., 147 2002) using LR-reactions generated the expression vectors pExp-Nhalo-cnx7, pExp-Chalo-cnx6 148 and pExp-Chalo-map65. 
All BiFC expression vectors and entry vectors with coding sequences of Cnx1 (AT5G20990), LA (Lifeact; amino acids 1-17 of the Saccharomyces cerevisiae protein ABP140), ABD2

152 available and are described by Kaufholdt et al. (2016a). The entry vectors were used to clone

153 Split-HaloTag ${ }^{\circledR}$ expression vectors via LR-reactions into pDest-GW-Nhalo and pDest-GW-Chalo

154 to create pExp-cnx1-Nhalo, pExp-Ia-Nhalo, pExp-ckl6-Nhalo, pExp-la-Chalo, pExp-abd2-Chalo 155 and pexp-Nluc-Chalo.

\section{Plant transformation}

$157 \quad$ N. benthamiana wild type plants were cultivated in soil under greenhouse conditions. They 158 were used for Agrobacterium-mediated transient transformation of fusion constructs 7 to 12 159 weeks after germination as described by Gehl and colleagues (2011). Agrobacterium strain $160 \mathrm{C} 58 \mathrm{C} 1 / \mathrm{pMP} 90$ carrying binary expression vectors were freshly grown $\left(48 \mathrm{~h}\right.$ at $28^{\circ} \mathrm{C}$ ) on solid $161 \mathrm{CPY}$ media $(0.1 \%(\mathrm{w} / \mathrm{v})$ yeast extract, $0.5 \%(\mathrm{w} / \mathrm{v})$ casein peptone, $0.5 \%(\mathrm{w} / \mathrm{v})$ sucrose, $2 \mathrm{mg} / \mathrm{L}$ $162 \mathrm{MgSO}_{4} \times 7 \mathrm{H}_{2} \mathrm{O}(\mathrm{pH} \mathrm{7}) ; 1.5 \% \mathrm{w} / \mathrm{v}$ agar) containing rifampicin (50 mg/L) and gentamycin 163 (50 mg/L) as well as kanamycin (50 mg/L). Helper strain p19 (Voinnet et al.,2003) was grown on CPY medium containing rifampicin (50 mg/L) and kanamycin (50 mg/L). After growing for $20 \mathrm{~h}$ in $9 \mathrm{~mL}$ of liquid CPY at $200 \mathrm{rpm}$ at $28^{\circ} \mathrm{C}$, cells transferred into fresh activation medium (10 mM MES/KOH (pH 5.6), $10 \mathrm{mM} \mathrm{MgCl}_{2}, 150 \mu \mathrm{M}$ acetosyringone). Before infiltration of the bacteria into the leaves, each strain was diluted in activation media to an optical density of $\mathrm{OD}_{600}=0.9$ (final $\mathrm{OD}_{600}=0.3$ ). Then, three strains were mixed for each transformation: (i) One strain containing a NHalo construct, (ii) one strain containing a CHalo-construct and (iii) the helper strain p19. After incubation for $2 \mathrm{~h}$ at $50 \mathrm{rpm}\left(28^{\circ} \mathrm{C}\right)$, mixed Agrobacterium suspension

171 were infiltrated into abaxial site of young but fully expanded leaves. Plants were incubated for $172 \quad 3-5$ days in the green house.

\section{Staining of $\boldsymbol{N}$. benthamiana leaves discs with HaloTag ${ }^{\circledR}$ ligands}

174 Staining protocol was based on the work of Lang and colleagues (2006). Leaf discs of (6$17510 \mathrm{~mm}$ ) of $N$. benthamiana leaves were transferred into a $20 \mathrm{~mL}$ syringe with screw lid and 176 infiltrated with 2-4 mL ligand solution $(0.5,1.0$ or $2.0 \mu \mathrm{M}$ TMR, DiAcFAM and Oregon Green in $17710 \mathrm{mM} \mathrm{MES} / \mathrm{KOH}(\mathrm{pH} 5.6)$ and $10 \mathrm{mM} \mathrm{MgCl}_{2}$ ). All dyes were purchased from Promega 178 (https://www.promega.de/). Syringes with leaf discs were wrapped in aluminium foil and 
179 incubated for $0.5,15,30$ or 60 min either on the work bench, on a tumbling shaker or on a 180 rotary tube mixer. After staining, the samples were washed with $10 \mathrm{~mL}$ washing solution by 181 closing the screw lid and moving the plunger up and down for 10 times. Washing steps were 182 repeated with fresh washing solution 6-12 times. Furthermore, one duration before the last 183 washing step of 0,3 or 12 hours in washing solution was performed.

\section{Confocal laser scanning microscopy}

185 The confocal Laser Scanning Microscope LSM 510Meta from Zeiss (Göttingen, Germany) was used. The cLSM-510META scanhead was connected to the Axiovert 200M. All images were examined using either the Plan-Neofluar $10 x / 0.3$ or the C-Apochromat $40 x / 1.2$ waterimmersion objective. For excitation, both an argon laser (488 nm for BiFC, Oregon Green and DiAcFAM as well as chlorophyll fluorescence) and a Helium-Neon Laser (543 nm line for TMR) was used. The emitted light passed the primary beam-splitting mirror UV/488/543/633 and was separated by a secondary beam splitter at $545 \mathrm{~nm}$. Fluorescence was detected with filter sets as follows: BP 505-530 nm for BiFC (Emmax: $515 \mathrm{~nm})$, Oregon Green (Emmax: $520 \mathrm{~nm})$ and

193 DiAcFAM (Emmax: $521 \mathrm{~nm}$ ); BP 560-615 for TMR (Emmax: $578 \mathrm{~nm}$ ); LP $650 \mathrm{~nm}$ for chlorophyll 194 fluorescence. Bright field images were taken with the transmitted light photomultiplier. All 195 images were taken using ZEISS Microscope Software ZEN 2009 and processed with ZEN lite 196 and Fiji (Schindelin et al., 2012). The shown images depict represented cells of several analysed leaves from at least three independent transformations.

\section{Super-resolution by polarisation demodulation microscopy}

199 Principles of the experimental set-up are described by Hafi and colleagues (2014) and 200 modifications enabling the analysis of the dye's 3D-orientations were described by Albrecht 201 et al. (2020). The cover slip was fixed to the microscope slide with nail polish. Linearly polarised 202 light deriving from a $488 \mathrm{~nm}$ continuous wave (CW) laser (sapphire 488-50, Coherent) was 203 used for excitation of Oregon Green molecules. The beam was expanded through a telescope 204 system. The polarisation was modulated at 15 frames per modulation period by rotation of a $205 \lambda / 2$-waveplate. The rotation was achieved through a chopper wheel (Optical Chopper 206 Systems, Thorlabs) which was synchronised to an electron-multiplying charge-coupled device 207 (EMCCD) camera (iXonEM+897 back illuminated, Andor Technology). Through the rotation of 
209 fluorophores being excited from a different direction. Then, the beam was focused onto the

210 back aperture of the microscope objective (UPlanSApo, 60x, NA $=1.35$ oil immersion,

211 Olympus), which was integrated in an inverted microscope body (IX 71, Olympus). Emitted

212 light was then passed through a dichroic mirror (beam splitter z 488 RDC, AHF) and an emission

213 filter (ET band pass 525/50, AHF). To further magnify the image and focus it the EMCCD

214 camera an additional lenses system was used. During the measurement 2,000 frames at

215 approximately $32 \mathrm{~ms}$ per frame were recorded. The first 200 frames were neglected for

216 calibration purposes. The raw fluorescence intensity of all modulation periods of the last 1,800

217 frames of a measurement was used for analyses. Images were "deblurred" by using

218 deconvolution algorithms. The "blurring" function or point-spread function (PSF) was

219 approximated by using the PSF-Generator Plugin for ImageJ

220 (http://bigwww.epfl.ch/algorithms/psfgenerator/). Using the PSF, the modulating

221 fluorescence intensities were deblurred using an iterative least-squares deconvolution while

222 accounting for the polarisation modulation. The least-squares functional was minimised by

223 using the FISTA Algorithm (Beck and Teboulle, 2009). 


\section{Results and Discussion}

225 The Split-HaloTag ${ }^{\circledR}$ constructs created in this study are based on the enhanced HaloTag ${ }^{\circledR}-7$ 226 sequence, which has been optimised and improved with regard to solubility, stability, binding 227 kinetics and access to an optional TEV-cleavage site (Ohana et al., 2009). According to the 228 initial experiment of Ischikawa and colleagues (2012) HaloTag ${ }^{\circledR}$ protein was split on position 229 155/156 aa into the N-terminal fragment "NHalo" (aa 1-155) and the C-terminal fragment 230 "CHalo" (aa 156-298). The stable bond between a HaloTag ${ }^{\circledR}$ protein and its ligand is formed 231 by the catalytic amino acid $\mathrm{Asp}^{106}$ as part of the "NHalo" fragment. In the wild type 232 dehalogenase, the closely located $\mathrm{His}^{272}$ would catalyse hydrolysis of the intermediate, 233 resulting in product release and enzyme regeneration (Los et al., 2008). In the mutated 234 HaloTag ${ }^{\circledR}$ protein, however, the substituted Asn ${ }^{272}$ (HaloTag ${ }^{\circledR}-7$ ) as part of "CHalo" traps the 235 reaction intermediate as a stable covalent adduct. Taking human embryonic kidney cells as a 236 model system, Ishikawa and colleagues (2012) affirmed the general capacity of Split-HaloTag ${ }^{\circledR}$ reconstitution by using a self-associating Split-GFP system. Furthermore, they monitored membrane fusion as cell fusion enabled functional HaloTag ${ }^{\circledR}$ reconstitution resulting in TMR signals after staining. In this method paper, we set out to (I) examine the utility of the SplitHaloTag ${ }^{\circledR}$ system as new tool to study protein-protein interactions in plant cells, (II) to develop staining and washing protocols resulting in low background fluorescence and (III) to demonstrate application examples of Split-HaloTag ${ }^{\circledR}$ for sophisticated imaging and advanced microscopy methods.

\section{Reconstitution of Split-HaloTag ${ }^{\circledR}$ in planta}

246 The cDNAs of the interaction partners were fused $\mathrm{N}$ - or C-terminally to the NHalo and CHalo 247 fragments via fusion PCR and gateway cloning (see Materials and Methods). For high 248 flexibility, Split-HaloTag ${ }^{\circledR}$ GATEWAY compatible destination vectors were generated which enabled a fast and easy cloning of expression vectors with coding sequences of different proteins of interest. The complex formation of the heterotetrameric molybdopterin synthase 251 (MPT) subunits $\mathrm{Cnx6}$ and $\mathrm{Cnx7}$ from Arabidopsis thaliana was used to demonstrate the 252 capability of reconstitution of the Split-HaloTag ${ }^{\circledR}$ in planta. This protein pair was chosen as 253 positive control due to their verified high binding strength (Kaufholdt et al., 2013). The two 
interacting proteins (Fig. 1A) will bring the two HaloTag ${ }^{\circledR}$ reporter fragments in close spatial proximity and, thereby, guide the reconstitution of functional HaloTag ${ }^{\circledR}$ proteins.

After transformation of Nicotiana benthamiana epidermis cells and staining with the fluorescent ligand TMR, specific cytosolic fluorescence was observed as a thin layer at the cell periphery (Fig. 1B). Wild type leaves were stained similarly, and lacking fluorescence signals indicated the washing steps being sufficient to remove unbound ligands (Fig. 1C). Therefore, since the MPT synthase complex is localised in the cytoplasm, HaloTag ${ }^{\circledR}$ fragments CHalo and NHalo were capable of reconstitution guided by the strong interaction of $\mathrm{Cnx} 6$ and $\mathrm{Cnx7}$. The reconstituted HaloTag ${ }^{\circledR}$ was able to bind red fluorescent ligand TMR (Em $\max 578 \mathrm{~nm}$ ), green fluorescent ligand Oregon Green (Em $\max 520$ nm) (Fig. 1D) as well as yellow fluorescent ligand DiAcFAM (Em $\max 526 \mathrm{~nm}$ ) (Fig. 1E), even though DiAcFAM signals were weaker compared to TMR and Oregon Green when stained with similar concentration. This demonstrates a general binding capability of the reconstituted HaloTag ${ }^{\circledR}$ exemplary for a plurality of other available dyes depending on the individual setting. As the two amino acids (Asp ${ }^{106}$ as part of NHalo and Asn ${ }^{272}$ as part of $\mathrm{CHalo}$ ) important for covalent ligand binding are located on each of the two separate Split-HaloTag ${ }^{\circledR}$ fragments, individual expression of "NHalo" or "CHalo" fragments will not enable ligand binding without each other which is a fundamental aspect when using SplitHaloTag ${ }^{\circledR}$ imaging for investigation of protein-protein interactions (Fig. 1F/G).

After proving the general reconstitution with a protein pair forming a permanent complex, this new assay was tested in a second approach with MPT synthase subunit Cnx6 and molybdenum insertase $\mathrm{Cnx1}$. In contrast to the permanent interactions within the MPT-

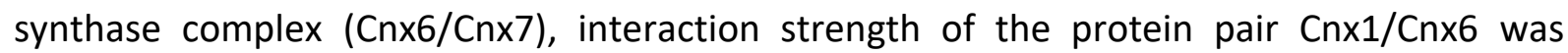
previously found to be distinct but of a more transient nature (Kaufholdt et al., 2013). Like in previous BiFC and Split-Luciferase experiments (Kaufholdt et al., 2013), we again conducted a full PPI study including all necessary controls. As control proteins, the cytosolic proteins NLuc (N-terminus of the luciferase from Photinus pyralis) and the G-box protein GF14 (AT1G78300) were provided and both represent proteins showing no interaction with $\mathrm{Cnx1}$ or $\mathrm{Cnx6}$. To ensure staining with equal TMR concentration and a similar washing procedure leaf discs of interaction approach and controls were punched in slightly different sizes and stained

283 simultaneously in the same syringe. The interaction approach showed strong cytosolic 
285 interactions. The negative control and both abundance controls showed weak cytosolic TMR

286 signals, too (Fig.S1B-D). A negative control without any fluorescence would be an unrealistic

287 event and observed spontaneous self-assembly was expected as it is typical for split-protein

288 assays such as BiFC and Split-Luciferase when proteins are overexpressed in the small cytosolic

289 space of plant cells (Gehl et al., 2009/2011). To evaluate whether differences in interaction

290 approach and negative control are due to different protein concentrations we previously

291 introduced additional abundance controls for our BiFC and Split-Luciferase studies (Kaufholdt

292 et al., 2016b). The amounts of expressed proteins were therefore similar in all approaches.

293 Taken all together, it can be concluded that random self-assembly can successfully be

294 distinguished from real interactions. In comparison, BiFC fragments have an intrinsic affinity

295 towards each other and once the BiFC complex is formed, this formation is irreversible

296 (Kerppola, 2008). Irreversibility can also be assumed for Split-HaloTag ${ }^{\circledR}$ since ligands will only

297 covalently bind to reconstituted HaloTag ${ }^{\circledR}$ proteins. However, with adequate negative

298 controls and a careful evaluation of obtained results, the spontaneous self-assembly of both

299 BiFC and Split-HaloTag ${ }^{\circledR}$ experiments can be overcome. Using the transient interaction of the

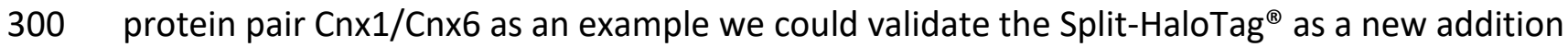

301 to the large toolbox for investigation of PPIs in planta. 


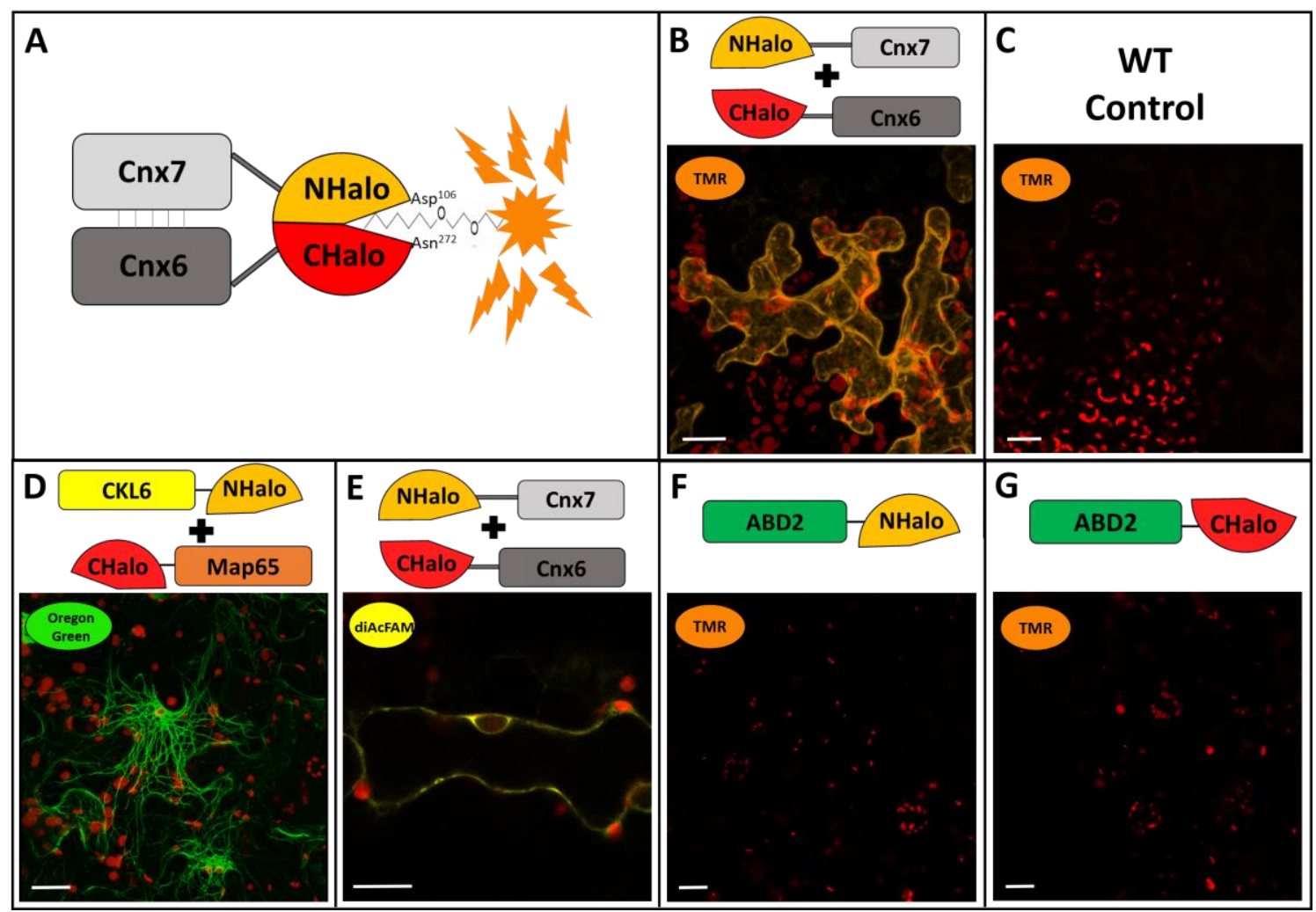

Fig. 1: Testing Split-HaloTag ${ }^{\circledR}$ complementation upon protein interactions of MPT synthase complex with different fluorescent ligands

Shown are images of $N$. benthamiana epidermis cells via confocal microscopy. Staining of leaf discs was performed 4-5 days after transformation. All images were taken with a C-Apochromat 40x/1.2 water immersion objective. Scale bars depict a length of $20 \mu \mathrm{m}$ each. (A) Schematic illustration of SplitHaloTag $^{\circledR}$ reconstitution guided by the MPT synthase subunits $\mathrm{Cnx6}^{6}$ and $\mathrm{Cnx7}$. The important amino Asp106 and Asn272acids of each reporter terminus for covalent linker binding are depicted. (B)

311 Cytosolic TMR fluorescence after transformation with NHalo-Cnx7 and Cnx6-CHalo. (C) Negative 312 control of a wildtype (WT) leaf after staining. (D) Oregon green fluorescence at microtubules filaments after transformation of microtubules binding constructs CKL6-NHalo and CHalo-Map65. (E) Cytosolic diAcFAM fluorescence after transformation with NHalo-Cnx7 and Cnx6-CHalo. (F/G) Negative controls of single transformed Split-HaloTag ${ }^{\circledR}$ reporter constructs fused to ABD2.

\section{Insights into the Staining Protocol}

317 Lang and colleagues (2006), who first introduced the HaloTag ${ }^{\circledR}$ system to plant cells, attributed 318 great importance to washing procedures to reduce unspecific background fluorescence as background-less staining is often more complicated in plants than in animals. For interaction studies, comparison of fluorescence intensity and fluorescence pattern is the main task, and 
322 protocol an excess of unbound dye remained in the tissue. Therefore, optimised staining and 323 destaining procedures had to be established for using Split-HaloTag ${ }^{\circledR}$ as reliable tool for PPI 324 studies. To improve staining protocols and to evaluate the best HaloTag ${ }^{\circledR}$ ligand several different factors were investigated: (i) the size of analysed leaf discs $(6 ; 8 ; 10 \& 12 \mathrm{~cm}$ in diameter), (ii) concentration of ligands $(0.25,0.5,1 \& 2 \mu \mathrm{M})$, (iii) ligand incubation time (1 min up to $1 \mathrm{~h}$ ), (iv) number of subsequent washing steps (6 up to 12), (v) incubation time in washing solution ( $3 \mathrm{~h}$ up to $12 \mathrm{~h}$ ) and (vi) aeration of leaf discs in washing solution.

During this optimisation process, several observations were made that are worth mentioning and need to be considered to prevent misinterpretation of gained results. After TMR staining, fluorescence was always detected in vascular tissue of transformed as well as wild type leaves suggesting a nonspecific adhesion between TMR and molecules in leaf veins (Fig. S2A). 333 Therefore, leaf area further away from vascular tissue should be used for analysis. 334 Furthermore, staining with more than $0.5 \mu \mathrm{M}$ TMR combined with an insufficient number of washing steps resulted in oversaturation and accumulation of unbound dye in the cytoplasm of parenchyma cells (Fig. S2B). This amount of unbound TMR accumulation increased when using larger or damaged leaf discs or older plants. Moreover, recycling of frozen TMR solution led to unspecific aggregations inside the cells.

DiAcFAM staining gained an overall weaker fluorescence signal compared to TMR but no staining of vascular tissue was observed (not shown). However, weak DiAcFAM signals (Emmax $521 \mathrm{~nm}$ ) could easily be mistaken for typical plant background fluorescence at approx. $342530 \mathrm{~nm}$. Furthermore, accumulation of unbound ligands occurred especially in stomata after 343 DiAcFAM (Fig. S2C) but also after Oregon Green (Fig. S2D) staining. In addition, Oregon Green resulted in accumulation inside vacuoles of parenchyma cells if incubated more than a few seconds in staining solution (Fig. S2E).

After testing of the different staining parameters optimal results for TMR staining were 347 obtained using leaf discs of a diameter of $6-8 \mathrm{~mm}$, a final TMR concentration of $0.5 \mu \mathrm{M} \mathrm{in} 2 \mathrm{~mL}$ 348 fresh staining solution, 15 min incubation time and followed by eight subsequent washing steps into a $20 \mathrm{~mL}$ syringe and an overnight incubation in washing solution. Samples were 350 incubated with $10 \mathrm{~mL}$ washing solution by closing the screw lid and moving the plunger up and down for approx. 10 times. Immediately before microscope analysis two more washing 
steps were performed. Both, overnight incubations either on a tumbling shaker or on a rotary

353 tube mixer were equally sufficient for all dyes, as long as there was sufficient air in the syringe

354 to allow leaf disc aeration. It must be noted that application of strong pressure during staining

355 and washing procedure can cause severe stress and damage to the cells. Therefore, pressure 356 to leaf discs in the syringe should be as low as possible but just enough for successful removal 357 of all unbound ligand.

Oregon Green showed optimal results if a $0.5 \mu \mathrm{M}$ staining solution was exchanged with $2 \mathrm{~mL}$ washing solution immediately after its infiltration and incubated for 15 minutes. Then, the washing procedure was applied as described for TMR. This could reduce but not completely avoid Oregon Green accumulation.

Overall, all exemplary tested dyes are suitable for intracellular cytosolic labelling of HaloTag ${ }^{\circledR}$ proteins in plant tissue, which leads to the assumption that other HaloTag ${ }^{\circledR}$ ligands will be suitable for other experimental settings as well. In this study, both TMR and Oregon Green showed ideal properties for confocal laser scanning microscopy despite Oregon Green accumulations. Due to a different experimental set-up only Oregon Green was used in SPoD microscopy. TMR showed the best applicability for usage within a Split-HaloTag ${ }^{\circledR}$ complementation assay for staining $N$. benthamiana leaf discs.

Validation of Split-HaloTag ${ }^{\circledR}$ imaging upon protein-protein interaction at cytoskeletal elements

371 To further prove the usability of Split-HaloTag ${ }^{\circledR}$ for in planta PPI studies, a third approach was conducted with proteins attached to cytoskeleton structures such as filamentous (F-) actin as

373 well as microtubules. By applying this approach, it was possible to test whether the Split374 HaloTag ${ }^{\circledR}$ system allows for investigating the assembly of different proteins at cytoskeletal elements. Both structures were not labelled directly to NHalo or CHalo termini, but via binding proteins, as a fusion of larger reporter fragments directly to globular actin or tubulin proteins

377 might disturb their polymerisation processes. For F-actin labelling, the binding domain of 378 fimbrin from A. thaliana (ABD2; Sheahan et al., 2004) as well as of Abp140 from 379 Saccharomyces cerevisiae (Lifeact/LA; Riedl et al., 2008) were used. Furthermore, microtubule 380 binding domains of the two proteins Casein-Kinase-1-Like-6 (CKL6; Ben-Nissan et al., 2008) 
investigated. The cytoskeleton binding proteins show no direct protein interactions to each

other. However, their affinity and subsequent binding and anchoring to the cytoskeletal structures results in such spatial proximity that it is able to act as model for a direct interaction of a cytoskeleton associated protein complex (Kaufholdt et al., 2016a). Expression of F-actin binding protein constructs followed by HaloTag ${ }^{\circledR}$ reconstitution and staining resulted in a TMR specific fluorescence visible as transversely arranged filaments with branches distributed throughout the cytoplasm (Fig. 2A1). The approach with CLK6-NHalo and CHalo-Map65 to label microtubules upon Split-HaloTag ${ }^{\circledR}$ reconstitution (Fig. 1D/Fig. 2A2) displayed filamentous structures more equally distributed throughout the cell with less cross bridges compared to actin filaments. These structures are typical for F-actin and microtubules, respectively, and were observed in BiFC experiments before (Kaufholdt et al., 2016a). In all interaction approaches both actin filaments as well as microtubules, could successfully be visualised upon interaction of cytoskeletal binding proteins.

Then, we aimed to validate this new assay with a binding assay of the molybdenum insertase Cnx1 to F-actin as a well-characterised example for a protein-protein interaction study at cell structures (Kaufholdt et al. 2016a). This established setting via cytoskeleton binding proteins for in vivo interaction studies was used to investigate whether the Split-HaloTag ${ }^{\circledR}$ system demonstrate the F-actin binding of $\mathrm{Cnx} 1$ in the same manner it was shown by BiFC experiments before (Kaufholdt et al. 2016a). In the interaction approach, reporter fusion constructs of $\mathrm{Cnx} 1$ and the actin binding protein LA, respectively, were co-expressed in $N$. benthamiana. For comparison, the BiFC approach described by Kaufholdt et al. (2016a) was included, for which the reporter halves VYNE (N-terminus of Venus) and SCYCE (C-terminus of SCFP) were used (Gehl et al., 2009). Both BiFC and Split-HaloTag ${ }^{\circledR}$ complementation assay show almost identical results (Fig. 2B/C). Both TMR and GFP fluorescence were detected in a filamentous pattern concentrated at the actin nucleus basket and thinned out at F-actin towards the cellular cortex. A typical pattern for studying an actin interacting protein complex was observed in both approaches that is reminiscent of a "starry sky" (Kaufholdt et al., 2016a) caused by F-actin anchoring of the interacting proteins in close proximity to its synthesis by the two actin binding domains of LA and Cxn1. When LA-NHalo was co-expressed with NLuc-

411 CHalo in the negative control, TMR specific fluorescence at actin filaments was detected, too. observed (Fig. 2D1/D2). Consequently, identical results of both BiFC and Split-HaloTag ${ }^{\circledR}$ were 


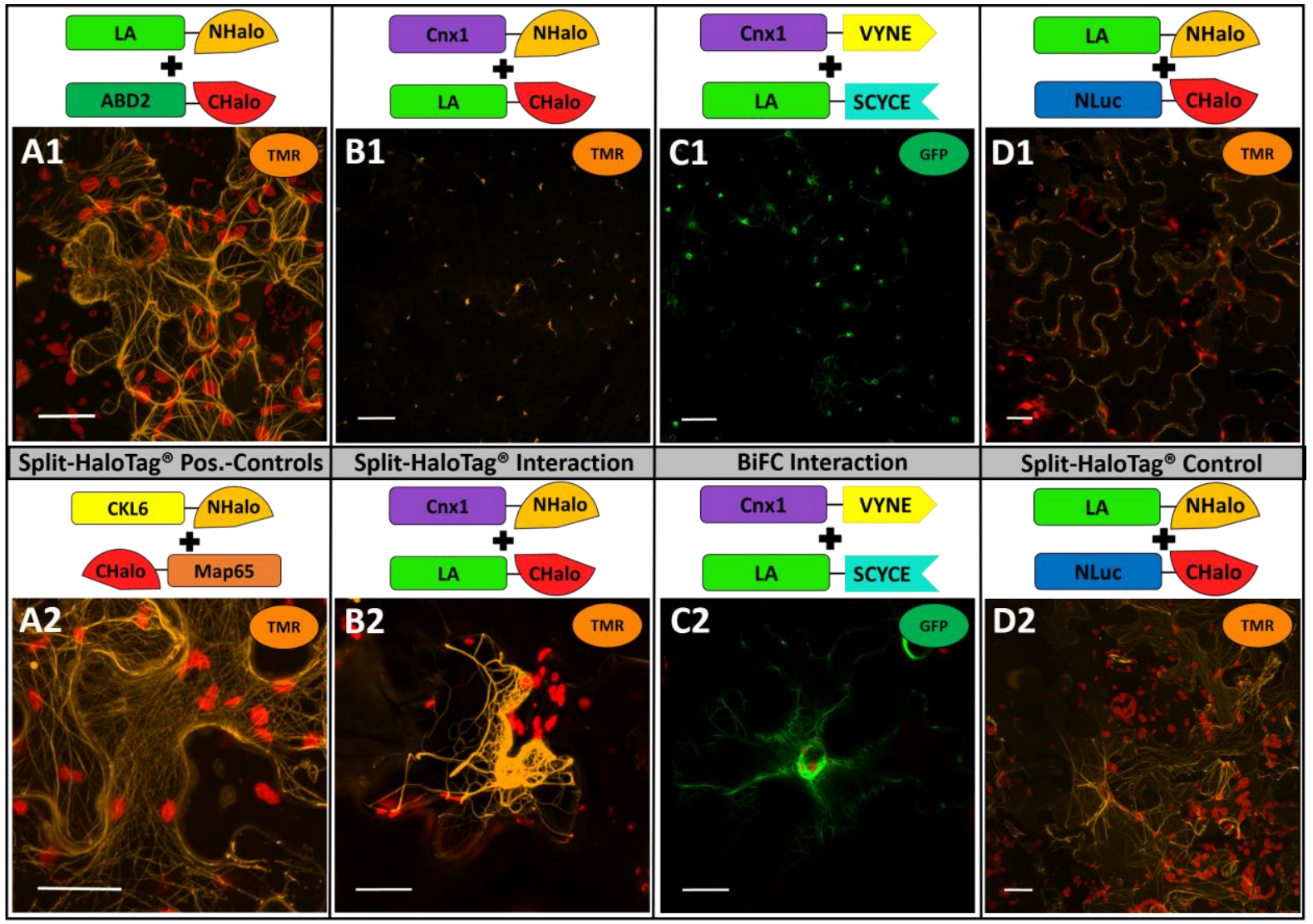

Fig. 2: Split-HaloTag ${ }^{\circledR}$ Protein-Protein Interaction Studies of $\mathrm{Cnx1}$ and Actin Filaments via Lifeact.

419 Shown are images of $N$. benthamiana epidermis cells via confocal microscopy of TMR or GFP. Staining

420 of leaf discs was performed 4-5 days after transformation. All images were taken with a Plan-Neofluar

421 10x/0.3 (B1/C1/D1) or with a C-Apochromat 40x/1.2 water immersion objective (A1/A2/B2/C2/D2).

422 Scale bars depict a length of $100 \mu \mathrm{m}$ (B1/C1/D1) or $20 \mu \mathrm{m}$ (A1/A2/B2/C2/D2). (A1) TMR fluorescence

423 at actin after transformation of actin binding constructs LA-NHalo and ABD2-CHalo. (A2) TMR

424 fluorescence at microtubules filaments after transformation of microtubules binding constructs CKL6-

425 NHalo and CHalo-Map65. (B) Split-HaloTag ${ }^{\circledR}$ approach with Cnx1-NHalo and LA-CHalo. (C) BiFC

426 approach with Cnx1-VYNE and LA-SCYCE. (D) Corresponding Split-HaloTag ${ }^{\circledR}$ negative control where

427 Cnx1 was replaced by the independent protein NLuc. (B) and (C) were imaged with identical setting for

428 optimal comparison of strength and pattern. 
Application examples of Split-HaloTag ${ }^{\circledR}$ for sophisticated imaging.

430 The Split-HaloTag ${ }^{\circledR}$ imaging assay has been proven as a feasible method for imaging of protein

431 interactions and obtained results of all given examples demonstrate similarity to BiFC results.

432 However, regarding protocol simplicity and handling, Split-HaloTag ${ }^{\circledR}$ imaging assay cannot

433 outcompete BiFC as method of choice for studying putative protein interaction. However, BiFC

434 is limited when confirmed specific protein interactions need to be observed and imaged with greater detail. As conventional light and fluorescence microscopy are diffraction-limited, advanced fluorescence imaging methods such as single molecule detection, subdiffractional polarisation imaging or super-resolution microscopy (SRM) techniques have been developed to improve the resolution and to allow studying molecular processes more detailed (Moerner and Kador 1989; Orrit and Bernard, 1990; Bode et al., 2008; Holleboom et al., 2014; Liao et al., 2010/2011; Godin et al., 2014; Loison et al., 2018; Camacho et al., 2019). For such imaging techniques, stable fluorescent dyes emitting light at various wavelength are needed which is hard to realise by standard fluorescent proteins (Banaz et al., 2018). When BiFC would be used to localise and image a specific protein interaction, detected fluorescence intensity would be directly interlinked with fusion construct expression levels. Compared to this, the SplitHaloTag ${ }^{\circledR}$ system has the advantage of adjusting the dosage of fluorescent dyes customised for the individual application. Using $0.25 \mu \mathrm{M}$ compared to $0.5 \mu \mathrm{M}$ for example enabled us to observe the attachment of molybdenum insertase $\mathrm{Cnx1}$ to F-actin with much greater detail (Fig. 3A/B). Using even lower concentrations would label even less HaloTag ${ }^{\circledR}$ proteins and enable tracking the dynamic movements of single protein complexes.

Especially during long-term observations, stability of fluorescent dyes is of great importance. Even though FPs have improved a lot in recent years with regard to their photon budget

452 (Kubitscheck et al., 2000) standard FPs used for most BiFC experiments show low quantum 453 efficiency, blinking behaviour and a high photobleaching rate (Reck-Petersen et al., 2006). In 454 a direct comparison, the GFP of the BiFC approach bleached much faster after 100 iteration 455 (100\% laser power) compared to TMR with same settings (Fig. 3B/C).

456 To show the stability and resolution potential of TMR for confocal laser scanning microscopy 457 the interaction of $C n \times 1$ and the actin binding protein ABD2 was again used as example. For this purpose, each layer of a cell needs to be scanned in very thin optical slices ( $\mu \mathrm{m}$ range or 
459 less) and such a detailed imaging can take several minutes. The stable TMR fluorescence of a 460 Split-HaloTag ${ }^{\circledR}$ enabled more defined results of cell images (Fig. 3D1) compared to BiFC 461 approaches (Fig. 3D2), which is demonstrated by the very thin filaments of the F-actin network 462 hardly detectable by BiFC.

463 Super-resolution by polarisation demodulation (SPoD) microscopy was used as second 464 example for demonstrating the performance of the Split-HaloTag ${ }^{\circledR}$ system. This advanced fluorescence imaging technique is a subdiffractional polarisation imaging method that allows measurement of the average orientation of fluorescent dyes attached to different structures and was first described by Hafi and colleagues (2014). Fluorescent molecules are illuminated via linearly polarised light. This causes the fluorophores to be excited at different times, which results in a modulated fluorescence intensity from the fluorophores. Depending on the orientation of the illuminated fluorophores (or more specifically the orientation of their transition dipole moments), the observed fluorescence intensity will be phase-shifted, and

472 differently oriented fluorophores will emit periodic signals peaking at different points in time. 473 Therefore, the analyses via deconvolution algorithms allow a high-resolution imaging of cell 474 structures (for details see Albrecht et al., 2020). During each measurement, 2,000 frames were 475 recorded, which in itself is not problematic when using stable dyes. Split-HaloTag ${ }^{\circledR}$ constructs 476 with the two microtubule binding domains of CLK6 and Map65 were used for this purpose. 477 Overexpression of MAP65 isotypes is known to result in microtubule bundling (Mao et al. 2006). After transformation and expression, leaf discs were stained with Oregon Green, which wavelength was more appropriate for SPoD microscopy. The observed fluorescence resulted in individual microtubules (Fig. 2E1). Albeit high amounts of background fluorescence and outof-focus signal complicated the recording and modulation analysis, subdiffractional separation 482 in a branching region of distinct fibres was observed in the deconvolved image (Fig. 3E2) and 483 was supported by different phases as visualised by a simple red/green colour code. These different phases in the branching region of the two fibres were already observable in the raw 485 modulation data. Certainly, future work is needed to enhance separation by different phases 486 in addition to pure image deconvolution for entire cell images. Nevertheless, Split-HaloTag ${ }^{\circledR}$ 487 imaging assay can be used for such advanced fluorescence imaging techniques, which failed 488 in case of BiFC. 


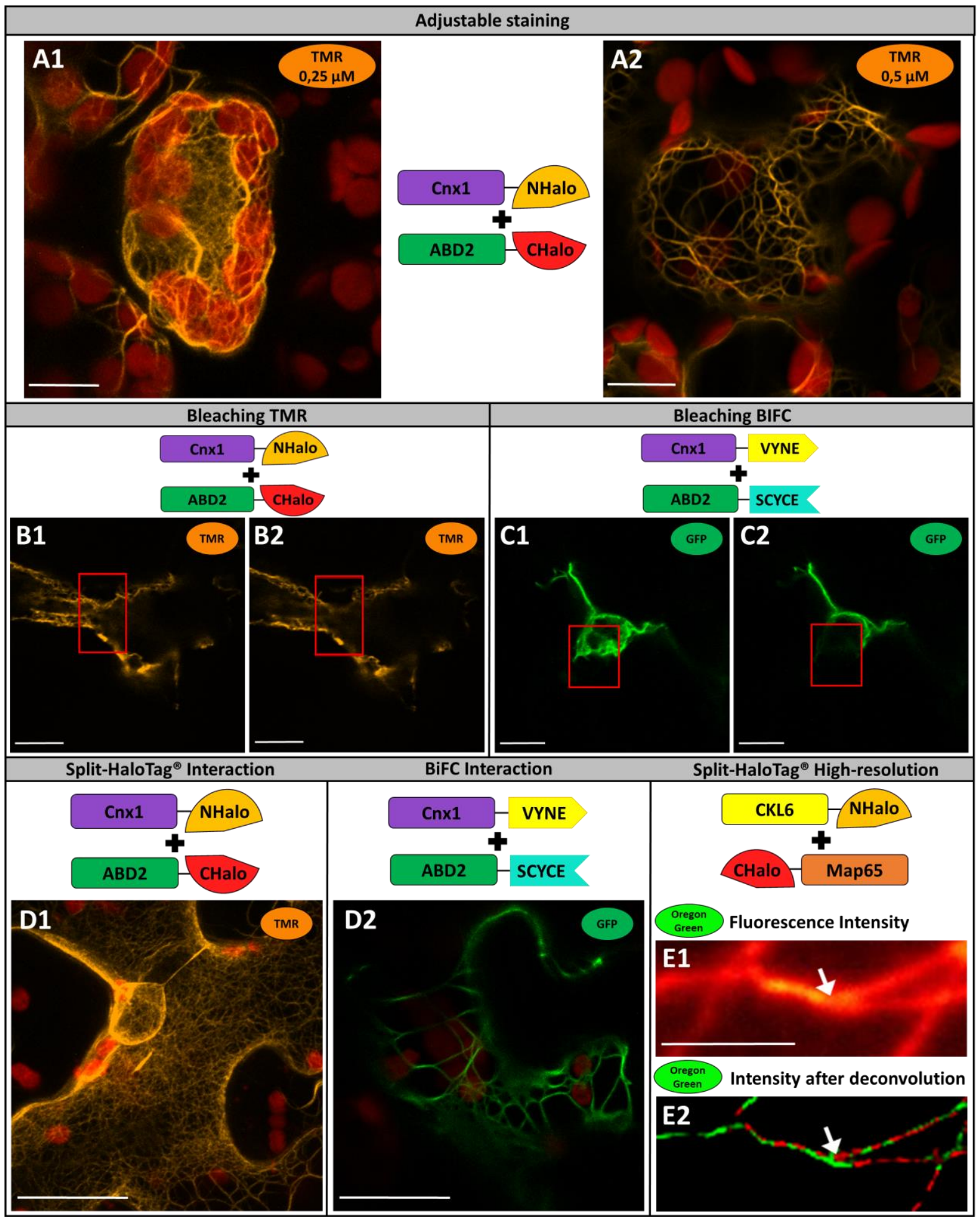

Fig. 3: Analysis of Split-HaloTag ${ }^{\circledast}$ images via confocal microscopy and super-resolution SPoD

491 microscopy.

492 Shown are representative $N$. benthamiana cells 4-6 days after transformation. (A) Staining with 493 different concentrations of TMR ((A1) 0,25 $\mu \mathrm{M}$ and (A2) 0,5 $\mu \mathrm{M})$ to optimise fluorescence intensity. 494 (B/C) Bleaching experiments of (B) TMR and (C) BiFC. Shown are pictures before (B1/C1) and after 495 (B2/C2) of 100 iterations of $100 \%$ laser power in the marked section (red rectangle). (D) Interaction 
studies at actin filaments with $\mathrm{Cnx1}$ and $\mathrm{ABD} 2$ via Split-HaloTag ${ }^{\circledR}$ (D1) or BiFC (D2). Split-HaloTag ${ }^{\circledR}$

497 Staining was performed with TMR. Images were taken with a C-Apochromat 40x/1.2 water immersion

498 objective. Scale bars depict a length of $20 \mu \mathrm{m}$. (E) SPoD microscopy of microtubules stained with

499 Oregon Green after transformation of the Split-HaloTag ${ }^{\circledR}$ microtubules binding constructs CKL6-NHalo

500 and CHalo-Map65. (E1) Diffraction limited image depicting averaged raw fluorescence intensity. (E2)

501 Phase colour coded fluorescence intensity image after 1000 iterations of the deconvolution algorithm.

502 The red/green colourcode support subdiffractional separation of the fibres at a distinct branching fork

503 (arrow) that is not visible in the conventional diffraction of wide field image. However, future work is

504 needed to enhance separation by different phases in addition to pure image deconvolution for entire

505 cell images. The raw data scale bars depict $2 \mu \mathrm{m}$ each.

\section{Conclusion}

507 In this study, Split-HaloTag ${ }^{\circledR}$ imaging assay was established for the first time in planta. Vectors 508 were cloned and reporter termini $\mathrm{NHalo}$ and $\mathrm{CHalo}$ were tested for reconstitution in both 509 fusion orientations to the protein of interest and in all four orientation combinations to each 510 other. The applicability of the system for protein-protein interaction studies was 511 demonstrated using previously published protein interactions forming the Molybdenum cofactor biosynthesis complex including its anchoring to F-actin. As TMR also penetrates 513 peroxisomal membranes (Lang et al., 2006) as well as the nuclear envelope (unpublished 514 data), interaction studies in other organelles would also be possible. Regarding protocol simplicity and handling, Split-HaloTag ${ }^{\circledR}$ imaging assay cannot outcompete BiFC as method of 516 choice for studying putative protein interactions. An additional infiltration of a fluorescent 517 ligand into the cell with subsequent washing steps was a disadvantage compared to BiFC. 518 However, relating to the background, Split-HaloTag ${ }^{\circledR}$ shows the same performance as BiFC, as 519 spontaneous self-assembly is typical for Split-protein assays when proteins are overexpressed 520 in the small cytosolic space of plant cells. The benefit of the Split-HaloTag ${ }^{\circledR}$ system lies in the 521 ability to visualise confirmed specific protein interactions with advanced imaging techniques.

522 Therefore, this system can be used in future for sophisticated imaging techniques such as 3D523 microscopy, polarisation-microscopy, single-molecule tracking or super-resolution imaging 524 methods that require brighter and more stable fluorescent markers. Localisation of protein 525 complexes can be observed with the Split-HaloTag ${ }^{\circledR}$ imaging assay in a distinct manner. In live526 cell microscopy, the method combines in vivo split-reporter analyses with the previous shown 527 advantages of the HaloTag ${ }^{\circledR}$ like a large set of differently coloured fluorescent ligands, their 
528 photostability compared to fluorescent proteins and the ability to vary labelling intensity via

529 adjusting the dosage of dyes independent from protein expression. In recent years, improved

530 FPs have already been used for split-reporter applications (Xie et al., 2017), however, some

531 drawbacks still remain. Therefore, this Split-HaloTag ${ }^{\circledR}$ imaging assay provides a unique and

532 sensitive approach for characterization of PPIs by combining all advantages given by the

533 HaloTag ${ }^{\circledR}$ system with the advantages of protein fragment complementation assays.

\section{Supplemental information}

535 Supplemental data are available online.

536 Figure S1: Split-HaloTag ${ }^{\circledR}$ Protein-Protein Interaction Studies of Cnx1 and Cnx6 analogue to 537 the BiFC study in Kaufholdt et al. (2013).

538 Figure S2: Evaluation of HaloTag ${ }^{\circledR}$ fluorescent Ligands TMR, DiAcFAM and Oregon Green.

539 Table S1: Primers for Cloning and sequencing of Split-HaloTag ${ }^{\circledR}$ related constructs and vectors.

540 Table S2: Split-HaloTag ${ }^{\circledR}$ Destination and Expression vectors used within this work.

\section{Acknowledgement}

543 We are grateful to Dr. Christin-Kirsty Baillie for scientific support and critical reading. We thank 544 Tanja Linke for excellent technical work in our lab. This work was financially supported by the 545 Deutsche Forschungsgemeinschaft (grant GRK2223/1) to RH and RRM.

\section{Author Contribution}

547 RMM, HB, PJW, RRM, RH and DK planned and designed the project. RMM, JNW, MB, CT, SF, 548 JS and DK cloned the vectors, performed plant transformation and the cLSM experiments. AA 549 and RM handled SPoD microscopy. All authors analysed and discussed the results. RMM, JNW, $550 \mathrm{RH}$ and DK were primarily involved in drafting the manuscript and RMM and DK produced 551 figures and tables. JS, HR, PJW and RRM critically read the manuscript and improved the text, 552 all authors finalised it. RH and DK coordinated the work. 
553

554

555

556

557

558

559

560

561

562

563

564

565

566

567

568

569

570

571

\section{References}

Albrecht, A., Pfennig, D., Nowak, J., Matis, R., Schaks, M., Hafi, N., Rottner, K., Walla, P. J. (2020). Amplitude Analysis of Polarization Modulation Data and 3D-Polarization Demodulation (3D-SPoD). bioRxiv. https://doi.org/10.1101/2020.03.10.986034.

Banaz, N., Mäkelä, J., Uphoff, S. (2018). Choosing the right label for single-molecule tracking in live bacteria: side-by-side comparison of photoactivatable fluorescent protein and Halo tag dyes. Journal of physics D: Applied physics, 52(6), 064002.

Beck, A., Teboulle, M. (2009). A fast iterative shrinkage-thresholding algorithm with application to wavelet-based image deblurring. In 2009 IEEE International Conference on Acoustics, Speech and Signal Processing, pp. 693-696.

Ben-Nissan, G., Cui, W., Kim, D. J., Yang, Y., Yoo, B. C., Lee, J. Y. (2008). Arabidopsis casein kinase 1like 6 contains a microtubule-binding domain and affects the organization of cortical microtubules. Plant physiology, 148(4), 1897-1907.

Bhat, R. A., Lahaye, T., Panstruga, R. (2006). The visible touch: in planta visualization of protein-protein interactions by fluorophore-based methods. Plant methods, 2(1), 12.

Bode, S., Quentmeier, C. C., Liao, P. N., Barros, T., Walla, P. J. (2008). Xanthophyll-cycle dependence of the energy transfer between carotenoid dark states and chlorophylls in NPQ mutants of living plants and in LHC II. Chemical Physics Letters, 450(4-6), 379-385.

Camacho, R., Täuber, D., Scheblykin, I. G. (2019). Fluorescence Anisotropy Reloaded-Emerging Polarization Microscopy Methods for Assessing Chromophores' Organization and Excitation Energy Transfer in Single Molecules, Particles, Films, and Beyond. Advanced Materials, 31(22), 1805671.

Cremer, C., Masters, B. R. (2013). Resolution enhancement techniques in microscopy. The European Physical Journal H, 38(3), 281-344.

England, C. G., Luo, H., Cai, W. (2015). HaloTag technology: a versatile platform for biomedical applications. Bioconjugate chemistry, 26(6), 975-986.

Gehl, C., Waadt, R., Kudla, J., Mendel, R. R., Hänsch, R. (2009). New GATEWAY vectors for high throughput analyses of protein-protein interactions by bimolecular fluorescence complementation. Molecular plant, 2(5), 1051-1058. 
581 Gehl, C., Kaufholdt, D., Hamisch, D., Bikker, R., Kudla, J., Mendel, R. R., Hänsch, R. (2011).

582 Quantitative analysis of dynamic protein-protein interactions in planta by a floated-leaf luciferase

583 complementation imaging (FLuCl) assay using binary Gateway vectors. The Plant Journal, 67(3), 542-

584553.

585 Godin, A. G., Lounis, B., Cognet, L. (2014). Super-resolution microscopy approaches for live cell 586 imaging. Biophysical journal, 107(8), 1777-1784.

587 Grimm, J. B., English, B. P., Chen, J., Slaughter, J. P., Zhang, Z., Revyakin, A., Patel, R., Macklin, J. J., 588 Normanno, D., Singer, R. H., Lionnet, T., Lavis, L. D. (2015). A general method to improve fluorophores 589 for live-cell and single-molecule microscopy. Nature methods, 12(3), 244.

590 Hafi, N., Grunwald, M., Van Den Heuvel, L. S., Aspelmeier, T., Chen, J. H., Zagrebelsky, M., Schütte, 591 O. M., Steinem, C., Korte, M., Munk, A., Walla, P. J. (2014). Fluorescence nanoscopy by polarization 592 modulation and polarization angle narrowing. Nature methods, 11(5), 579.

593 Hamada, T. (2007). Microtubule-associated proteins in higher plants. Journal of plant research, 120(1), 594 79-98.

595 Holleboom, C. P., Walla, P. J. (2014). The back and forth of energy transfer between carotenoids and 596 chlorophylls and its role in the regulation of light harvesting. Photosynthesis research, 119(1-2), 215597221.

598 Huang, B., Bates, M., Zhuang, X. (2009). Super-resolution fluorescence microscopy. Annual review of 599 biochemistry, 78, 993-1016.

600 Ishikawa, H., Meng, F., Kondo, N., Iwamoto, A., Matsuda, Z. (2012). Generation of a dualfunctional 601 split-reporter protein for monitoring membrane fusion using self-associating split GFP. Protein 602 Engineering, Design \& Selection, 25(12), 813-820.

603 Karimi, M., Inzé, D., Depicker, A. (2002). GATEWAY' ${ }^{\mathrm{TM}}$ vectors for Agrobacterium-mediated plant 604 transformation. Trends in plant science, 7(5), 193-195.

605 Kaufholdt, D., Gehl, C., Geisler, M., Jeske, O., Voedisch, S., Ratke, C., Bollhöner, B., Mendel, R. R., 606 Hänsch, R. (2013). Visualization and quantification of proteininteractions in the biosynthetic pathway 607 of molybdenum cofactor in Arabidopsis thaliana. J. Exp. Bot. 64, 2005-2016. 

filaments via molybdenum insertase $\mathrm{Cnx1}$ as anchor protein in Arabidopsis thaliana. Plant Science, 244, 8-18.

Kaufholdt, D., Baillie, C. K., Meyer, M. H., Schwich, O. D., Timmerer, U. L., Tobias, L., van Thiel, D.,

Kerppola, T. K. (2013). Bimolecular fluorescence complementation (BiFC) analysis of protein interactions in live cells. Cold Spring Harbor Protocols, 2013(8), pdb-prot076497.

Kubitscheck, U., Kückmann, O., Kues, T., Peters, R. (2000). Imaging and tracking of single GFP molecules in solution. Biophysical Journal, 78(4), 2170-2179.

Kudla, J. \& Bock, R. (2016). Lighting the way to protein-protein interactions: recommendations on best practices for bimolecular fluorescence complementation analyses. The Plant Cell, 28(5), 1002-1008. in plant cells. Journal of experimental botany, 57(12), 2985-2992. proteins in fixed and living cells using photoactivatable organic fluorophores. Journal of the American Chemical Society, 132(43), 15099-15101.

Liao, P.N., Bode, S., Wilk, L., Hafi, N., Walla, P. J. (2010). Correlation of electronic carotenoidchlorophyll interactions and fluorescence quenching with the aggregation of native LHC II and chlorophyll deficient mutants. Chemical Physics, 373(1-2), 50-55.

633 Liao, P. N., Pillai, S., Gust, D., Moore, T. A., Moore, A. L., Walla, P. J. (2011). Two-Photon Study on the 634 Electronic Interactions between the First Excited Singlet States in Carotenoid- Tetrapyrrole Dyads. The Journal of Physical Chemistry A, 115(16), 4082-4091. 
636 Liss, V., Barlag, B., Nietschke, M., Hensel, M. (2015). Self-labelling enzymes as universal tags for 637 fluorescence microscopy, super-resolution microscopy and electron microscopy. Scientific reports, 5, 63817740.

639 Loison, O., Weitkunat, M., Kaya-Çopur, A., Alves, C. N., Matzat, T., Spletter, M. L., Luschnig, S., 640 Brasselet, S., Lenne, P. F., Schnorrer, F. (2018). Polarization-resolved microscopy reveals a muscle 641 myosin motor-independent mechanism of molecular actin ordering during sarcomere maturation. PLoS biology, 16(4), e2004718.

643 Los, G., Wood, K. (2007). The HaloTag: A novel technology for cell imaging and protein analysis. 644 Methods. Mol. Biol. 356, 195-208.

645 Los, G. V., Encell, L. P., McDougall, M. G., Hartzell, D. D., Karassina, N., Zimprich, C., Wood, M. G., 646 Learish, R., Ohana, R. F., Urh, M., Simpson, D., Mendez, J., Zimmermann, K., Otto, P., Vidugiris, G., 647 Zhu, J., Darzins, A., Klaubert, D. H., Bulleit, R. F., Wood, K. V. (2008). HaloTag: a novel protein labeling 648 technology for cell imaging and protein analysis. ACS chemical biology, 3(6), 373-382.

Mao, G., Buschmann, H., Doonan, J. H., Lloyd, C. W. (2006). The role of MAP65-1 in microtubule bundling during Zinnia tracheary element formation. Journal of cell science, 119(4), 753-758.

651 Miyawaki, A., Sawano, A., Kogure, T. (2003). Lighting up cells: labelling proteins with fluorophores. Nat Cell Biol., S1-S7.

Moerner, W. E., Kador, L. (1989). Optical detection and spectroscopy of single molecules in a solid. 654 Physical review letters, 62(21), 2535.

655 Morisaki, T., McNally, J. G. (2014). Photoswitching-Free FRAP Analysis with a Geneticall Encoded 656 Fluorescent Tag. PLoS ONE, 9(9), e107730 a genetically engineered tag that enhances bacterial expression of soluble proteins and improves protein purification. Protein expression and purification, 68(1), 110-120. terphenyl crystal. Physical review letters, 65(21), 2716. molecule analysis of dynein processivity and stepping behavior. Cell, 126(2), 335-348. 
Riedl, J., Crevenna, A. H., Kessenbrock, K., Yu J. H., Neukirchen, D., Bista, M., Bradke, F., Jenne, D.,

665 Holak, T. A., Werb, Z., Sixt, M., Wedlich-Soldner, R. (2008). Lifeact: a versatile marker to visualize F666 actin. Nature methods, 5(7), 605.

667 Schindelin, J., Arganda-Carreras, I., Frise, E., Kaynig, V., Longair, M., Pietzsch, T., Preibisch, S., 668 Rueden, C., Saalfeld, S., Schmid, B., Tinevez, J. Y., White, D. J., Hartenstein, V., Eliceiri, K., Tomancak, 669 P., Cardona, A. (2012). Fiji: an open-source platform for biological-image analysis. Nature methods, 670 9(7), 676-682.

671 Sheahan, M. B., Staiger, C. J., Rose, R. J., \& McCurdy, D. W. (2004). A green fluorescent protein fusion 672 to actin-binding domain 2 of Arabidopsis fimbrin highlights new features of a dynamic actin 673 cytoskeleton in live plant cells. Plant physiology, 136(4), 3968-3978.

674 Struk, S., Jacobs, A., Sánchez Martín-Fontecha, E., Gevaert, K., Cubas, P., \& Goormachtig, S. (2019). 675 Exploring the protein-protein interaction landscape in plants. Plant, cell \& environment, 42(2), 387676409.

677 Voinnet, O., Rivas, S., Mestre, P., Baulcombe, D. (2003). An enhanced transient expression system in 678 plants based on suppression of gene silencing by the $\mathrm{p} 19$ protein of tomato bushy stunt virus. Plant J. $67933,949-956$.

680 Xie, W., Nielsen, M. E., Pedersen, C., Thordal-Christensen, H. (2017). A split-GFP gateway cloning 681 system for topology analyses of membrane proteins in plants. PloS one, 12(1), e0170118.

682 Zitnik, M., Feldman, M. W., Leskovec, J. (2019). Evolution of resilience in protein interactomes across 683 the tree of life. Proceedings of the National Academy of Sciences, 116(10), 4426-4433 